\title{
Draft
}

\section{Path and cycle factors of cubic bipartite graphs}

\author{
M. Kano ${ }^{1}$, Changwoo Lee ${ }^{2}$ and Kazuhiro Suzuki ${ }^{1}$ \\ ${ }^{1}$ Department of Computer and Information Sciences \\ Ibaraki University, Hitachi 316-8511 Japan \\ e-mail: kano@mx.ibaraki.ac.jp and tutetuti@dream.com; \\ ${ }^{2}$ Department of Mathematics \\ University of Seoul, Dongdaemoonku, Seoul 130-743, Korea \\ Dedicated to Professor Hikoe Enomoto on his 60th Birthday
}

\begin{abstract}
For a set $\mathcal{S}$ of connected graphs, a spanning subgraph $F$ of a graph is called an $\mathcal{S}$ factor if every component of $F$ is isomorphic to a member of $S$. It was recently shown that every 2-connected cubic graph has a $\left\{C_{n} \mid n \geq 4\right\}$-factor and a $\left\{P_{n} \mid n \geq 6\right\}$ factor, where $C_{n}$ and $P_{n}$ denote the cycle and the path of order $n$, respectively (Kawarabayashi et al., J. Graph Theory, Vol. 39 (2002) 188-193). In this paper, we show that every connected cubic bipartite graph has a $\left\{C_{n} \mid n \geq 6\right\}$-factor, and has a $\left\{P_{n} \mid n \geq 8\right\}$-factor if its order is at least 8 .
\end{abstract}

Key Words: Cycle factor, Path factor, bipartite graph,

MSC: 05C38, 05C70

\section{Introduction}

We consider finite graphs without loops or multiple edges. A 3-regular graph is called a cubic graph. We denote by $P_{n}$ and $C_{n}$ the path and the cycle of order $n$, respectively. For a set $\mathcal{S}$ of connected graphs, a spanning subgraph $F$ of a graph $G$ is called an $\mathcal{S}$-factor of $G$ if every component of $F$ is isomorphic to one of members in $S$. Then a $\left\{C_{n} \mid n \geq 3\right\}$-factor is nothing but a 2-factor, which is a spanning 2-regular subgraph.

In this paper we consider cycle-factors and path-factors of cubic graphs, whose components are cycles and paths, respectively. Notice that in a cubic graph, the edgeconnectivity is equal to the connectivity. We begin with some known results on these factors.

Theorem 1 (Petersen [5]) Every 2-connected cubic graph has a $\left\{C_{n} \mid n \geq 3\right\}$-factor. 
Kaneko found a criterion for a graph to have a $\left\{P_{n} \mid n \geq 3\right\}$-factor, and obtained the following theorem as its corollary. Note that a short proof of Kaneko's theorem can be found in [3].

Theorem 2 (Kaneko [2]) Every connected cubic graph has a $\left\{P_{n} \mid n \geq 3\right\}$-factor.

Recently Kawarabayashi et al [4] showed the next theorem.

Theorem 3 [4] (i) Every 2-connected cubic graph has a $\left\{C_{n} \mid n \geq 4\right\}$-factor.

(ii) Every 2-connected cubic graph of order at least six has a $\left\{P_{n} \mid n \geq 6\right\}$-factor.

In this paper we shall prove the following theorem.

Theorem 4 (i) Every connected cubic bipartite graph has a $\left\{C_{n} \mid n \geq 6\right\}$-factor.

(ii) Every connected cubic bipartite graph of order at least eight has a $\left\{P_{n} \mid n \geq 8\right\}$-factor.

We now give some remarks on the above Theorem 4. It follows immediately from Theorem 4 that every connected cubic bipartite graph $G$ of order at most 16 has a Hamiltonian path since $G$ has a $\left\{C_{n} \mid n \geq 6\right\}$-factor, which consists of at most two components, and a graph consisting of two disjoint cycles and one edge joining them has a Hamiltonian path. It is not mentioned in [4] that the conclusion of Theorem 3 is best possible. However, we can easily find 2-connected cubic graphs having no $\left\{C_{n} \mid n \geq 5\right\}$-factors. An example of such a cubic graph is given in Figure 1 (a), and it has many triangles. So we might expect that a 2-connected triangle-free cubic graph has a $\left\{C_{n} \mid n \geq 5\right\}$-factor. But this is not true as shown in Figure 1 (b), which shows a 2-connected triangle-free cubic graph having no $\left\{C_{n} \mid n \geq 5\right\}$-factor. Moreover, Theorem 4 is sharp in the sense that there exists a connected cubic bipartite graph having no $\left\{C_{n} \mid n \geq 8\right\}$-factor as shown in Figure 1 (c).

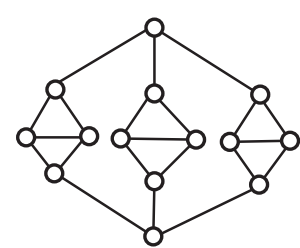

(a)

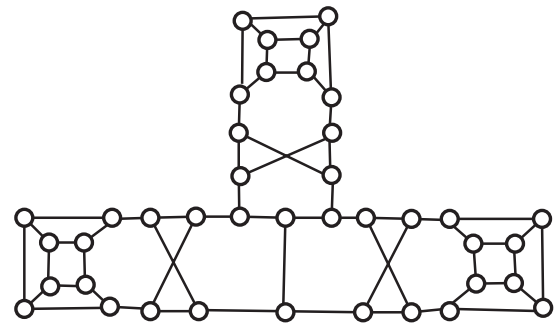

(b)

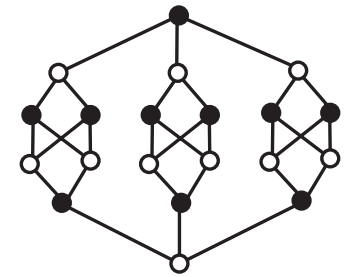

(c)

Figure 1: (a) A 2-connected cubic graph having no $\left\{C_{n} \mid n \geq 5\right\}$-factor; (b) A 2-connected triangle-free cubic graph having no $\left\{C_{n} \mid n \geq 5\right\}$-factor; (c) A 2-connected cubic bipartite graph having no $\left\{C_{n} \mid n \geq 8\right\}$-factor.

However we have been unable to find a 3-connected cubic graph having no $\left\{C_{n} \mid n \geq\right.$ $5\}$-factor or no $\left\{P_{n} \mid n \geq 7\right\}$-factor. So we propose the following conjecture and problem. 
Conjecture 5 Every 3-connected cubic graph of order at least six has a $\left\{C_{n} \mid n \geq 5\right\}$ factor.

Problem 6 Determine the maximum integer $k \geq 6$ for which every 3-connected (or 2connected) cubic graph of order at least $f(k)$ has a $\left\{P_{n} \mid n \geq k\right\}$-factor, where $f(k)$ is a suitable function of $k$.

We conclude this section with a conjecture on path-factors of 3-connected cubic graphs.

Conjecture 7 (Akiyama and Kano [1]) Every 3-connected cubic graph of order $3 n$ has a $\left\{P_{3}\right\}$-factor.

\section{Proof of Theorem 4}

For a vertex $v$ of a graph $G$, we denote by $\operatorname{deg}_{G}(v)$ the degree of $v$ in $G$. For two disjoint vertex subsets $X$ and $Y$ of $V(G)$, we denote by $e_{G}(X, Y)$ the number of edges of $G$ joining $X$ to $Y$. We denote the order of $G$ by $|G|$, which is equal to $|V(G)|$.

Lemma 8 Let $r \geq 2$ be an integer. Then every connected $r$-regular bipartite graph is 2-edge connected. In particular, every connected cubic bipartite graph is 2-connected.

Proof. Let $G$ be a connected $r$-regular bipartite graph with bipartition $A \cup B$. Suppose that $G$ has an bridge $e=u w \in E(G), u \in A, w \in B$. Then for a component $D$ of $G-e$ containing $u$ but not $w$, we have

$$
r|V(D) \cap A|-1=\sum_{x \in V(D) \cap A} \operatorname{deg}_{D}(x)=\sum_{x \in V(D) \cap B} \operatorname{deg}_{D}(x)=r|V(D) \cap B| .
$$

This is a contradiction. Hence $G$ has no bridge, which implies that $G$ is 2-edge connected.

We first prove (i) of Theorem 4 .

Proof of (i). Let $G$ be a connected cubic bipartite graph. We prove (i) by induction on the order $|G|$. There exists only one connected cubic bipartite graph of order six, which is $K_{3,3}$, and it has a $\left\{C_{6}\right\}$-factor. So we may assume $|G| \geq 8$.

By Lemma $8, G$ is 2-connected, and so $G$ has a 2 -factor $F$ by Theorem 1, which is a $\left\{C_{n} \mid n \geq 4\right\}$-factor. We may assume that $F$ contains a component $D$ isomorphic to $C_{4}$ since otherwise $F$ is the desired $\left\{C_{n} \mid n \geq 6\right\}$-factor. Let $V(D)=\{a, b, c, d\}$, and $a s, b t, c u, d w$ be the edges of $G-E(D)$ incident with $V(D)$ (see Figure 2).

Since $G-E(F)$ is a 1-factor of $G,\{a s, b t, c u, d w\}$ is a set of independent edges, and so $s, t, u, w$ are all distinct vertices of $G$. Let $H$ be the graph obtained from $G$ by removing the four vertices $a, b, c, d$ and their incident edges, and by adding two new vertices $x$ and $y$ together with five new edges $s x, u x, t y, w y, x y$ (see Figure 2). 

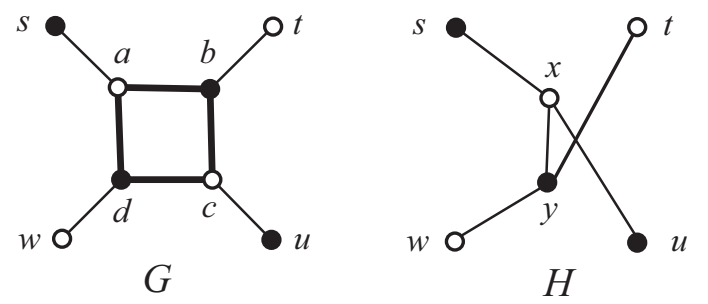

Figure 2: Cubic graphs $G$ and $H$; Bold lines are edges of $D$.

Then $H$ is a connected cubic bipartite graph, and $|H|=|G|-2$. Hence $H$ has a $\left\{C_{n} \mid n \geq 6\right\}$-factor $F_{H}$ by induction. We shall obtain the desired $\left\{C_{n} \mid n \geq 6\right\}$-factor of $G$ from $F_{H}$ by considering the following two cases.

Case 1. A component of $F_{H}$ contains the edge xy.

In this case, without loss generality, we may assume that a component $D$ of $F_{H}$ contains $x y, s x$ and $y w$ by symmetry. Then $F_{H}-\{s x, x y, y w\}+\{s a, a b, b c, c d, d w\}$ is the desired $\left\{C_{n} \mid n \geq 6\right\}$-factor of $G$.
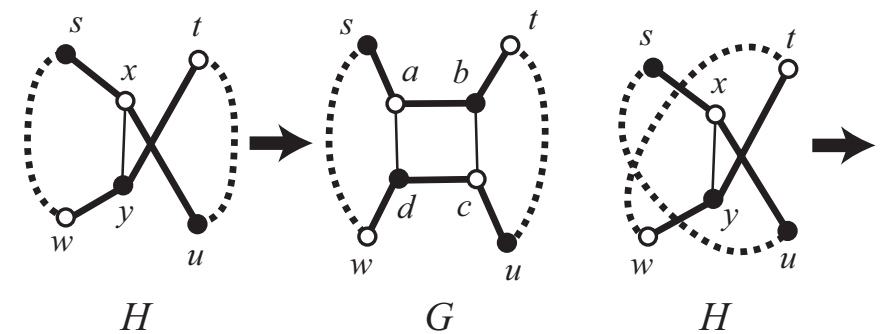

$H$

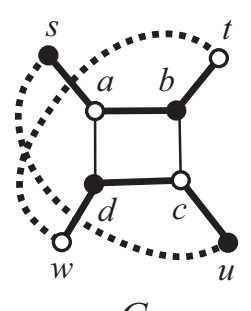

G

Figure 3: Cubic graphs $G$ and $H$ and their $\left\{C_{n} \mid n \geq 6\right\}$-factors.

Case 2. No component of $F_{H}$ contains the edge $x y$.

In this case $F_{H}$ contains the four edges $s x, x u, t y, y w$. We first assume that these four edges are contained in the same component $D$ of $F_{H}$. By symmetry, we may assume that a cycle $D$ passes through $s, x, u$ and then $t, y, w$ (see Figure 3 ). Then we can obtain the desired $\left\{C_{n} \mid n \geq 6\right\}$-factor from $F_{H}$ by removing the edges $s x, x u, t y, y w$ and by adding the edges $s a, a b, b t, u c, c d, d w$ as shown in Figure 3.

Next assume that the four edges $s x, x u, t y, y w$ are contained in two distinct components $D_{1}$ and $D_{2}$ of $F_{H}$. In this case we can obtain the desired $\left\{C_{n} \mid n \geq 6\right\}$-factor of $G$ from $F_{H}$ by removing $s x, x u, t y, y w$ and by adding $s a, a b, b t, w d, d c, c u$. Consequently Statement (i) of Theorem 4 is proved.

Statement (ii) of Theorem 4 follows immediately from the next Lemma 9 and the statement (i) of Theorem 4. It is shown in [4] that if a 2-connected cubic graph of order 
at least six has a $\left\{C_{n} \mid n \geq 4\right\}$-factor, then it has a $\left\{P_{n} \mid n \geq 6\right\}$-factor. This statement can be generalized as the following Lemma 9 without changing the proof.

Lemma 9 ([4]) Let $k \geq 3$ be an integer. If a 2-connected cubic graph $G$ of order at least $k+2$ has a $\left\{C_{n} \mid n \geq k\right\}$-factor, then $G$ has a $\left\{P_{n} \mid n \geq k+2\right\}$-factor.

\section{References}

[1] J. Akiyama and M. Kano, Path factors of a graph. Graphs and applications (Boulder, Colo., 1982), 1-21, Wiley-Intersci. Publ., Wiley, New York, 1985.

[2] A. Kaneko, A necessary and sufficient condition for the existence of a path factor every component of which is a path of length at least two. J. Combin. Theory Ser. B 88 (2003), 195-218.

[3] M. Kano, G.Y. Katona, Z. Kiraly, Packing paths of length at least two. Discrete Math. 283 (2004), 129-135.

[4] K. Kawarabayashi, H. Matsuda, Y. Oda and K. Ota, Path factors in cubic graphs. J. Graph Theory 39 (2002), 188-193.

[5] J. Petersen, Die Theorie der regulären Graphen, Acta Math. 15 (1891), 193-220. 